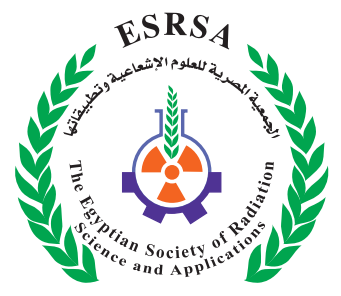

J. Nucl. Tech. Appl. Sci., Vol. 8, PP. 35 : 47 (2020)

\title{
Implementing Knowledge Management Program as a Part of Effective Integrated Management System for the ETRR2 Complex
}

Eisa, A.A. and Saleh, A.A.

Received: 15/12/2019

Accepted: 16/02/2020

E.mail:ayman_eisa@yahoo.com

\section{ABSTRACT}

In the IAEA documentations related to management, for example the recently issued IAEA Safety Requirements publication Leadership and Management for Safety (IAEA Safety Standards Series No. GSR Part 2), it is understood that the organization information and knowledge (both tacit and explicit) should be managed as a resource for effective implementation of the management system. Also, lack of a knowledge management program is one of the warning signs of a decline in safety culture. This paper focuses on enhancing the Knowledge Management performance as a main step to achieve the goal of applying the Integrated Management System to improve safety, operation efficiency and performance. The Egyptian Atomic Energy Authority (EAEA) is a governmental research authority that owns the Egypt Second Research Reactor (ETRR 2) complex. This Complex contains three nuclear installations (ETRR2 Reactor, Fuel Manufacturing Pilot Plant-FMPP and Radioisotopes Production

\section{KEYWORDS}

Data, Information, Knowledge

Management, Integrated Management System. Facility-RPF) and Administrative and engineering building (headquarter of the complex), which have a fairly adequate knowledge base. The paper introduces a proposal of Knowledge Management Program design for the ETRR2 complex. The objective of the proposed plan is first to evaluate the existing Knowledge base and then implement effective Knowledge Management (KM) program in order to avoid risks to overall operations such as 'Risk of Knowledge Loss' and lower safety and productivity.

1. Nuclear Safety and Radiological Emergency Department, National Center of Radiation Research and Technology (NCRRT), Egyptian Atomic Energy Authority (EAEA), Cairo, Egypt. 


\section{INTRODUCTION}



a) production changes,

b) plant growth and expansions,

c) replacement and renewal,

d) deterioration of primary equipment,

e) modifications to applicable laws, regulations, standards and codes, etc.

These aspects should be continually monitored and kept through effective knowledge management system to ensure that plant data and information for components and operation are adequately maintained overtime. Those circumstances, occurring individually or in combination, suggest that a knowledge management system is important to conduct necessary analysis and evaluation that may be required each time to ensure continued safe and reliable plant operation.

Also attrition, which is a decrease in the number of employees in an organization due to retirements, other terminations, or transfers to other organizations (brain drain), should be considered. In the nuclear industry attrition due to retirement is a particularly important issue because plants typically have stable workforces, all or most of whom joined during the commissioning phase, and thus they often have similar retirement dates. For this reason, explicit and tacit knowledge for staff should also be maintained overtime.

The Egyptian Atomic Energy Authority (EAEA) is a governmental research authority that owns the Egypt Second Research Reactor (ETRR 2) complex. This Complex includes the following main installa- tions at the Nuclear Research Centre of the (EAEA) in Inshas site:

1. A nuclear reactor (Egypt Second Research Reactor-ETRR2): used mainly for the generation and utilization of neutron flux and ionizing radiation for research and other purposes, including experimental facilities associated with the reactor,

2. A fuel manufacturing facility (Fuel Manufacturing Pilot Plant-FMPP): erected to assemble the fresh fuel elements that used for ETRR-2 core refueling,

3. A facility for producing radioisotopes (Radioisotopes Production Facility-RPF): erected to provide therapeutic and diagnosis isotopes to meet the requirements of Egyptian medical market and industrial radioisotopes to fulfill agricultural and industrial needs.

4. An administrative and engineering building (headquarter of the complex) contains: administrative offices, engineering laboratories, technical offices, stores, library and documents archives.

The paper focuses on enhancing the operating performance of the complex by introducing a proposal of Knowledge Management design as a main step to achieve the goal of applying the IMS (Integrated Management System) for the complex to improve safety, operation efficiency and performance.

\section{KNOWLEDGE MANAGEMENT AND ITS RELATION WITH INTERNATIONAL MANAGEMENT STANDARDS}

The following subsections show how the new international standards, related to management, consider that the organization information and knowledge (both tacit and explicit) should be managed as a resource for effective implementation of the management system. 


\section{ISO 9001:2015- fifth edition}

This new version of ISO standards pays more attention to information and knowledge. It contains an individual clause (no. 7.1.6), which consider the organizational knowledge as one of the support resources. It defines the organizational knowledge as the knowledge specific to the organization; which is generally gained by experience and it is information that is used and shared to achieve the organization's objectives. Also, as part of the alignment with other management system standards, a common clause on "documented information" has been adopted without significant change or addition (no. 7.5) (ISO 9000, 2015 - ISO 9001, 2015).

\section{Organizational Knowledge}

The new ISO standard addresses the need to determine and manage the knowledge maintained by the organization, to ensure the operation of its processes and that it can achieve conformity of products and services. Where, this knowledge is required to be maintained and be made available to the extent necessary. Also, when addressing changing needs and trends, the organization is required to consider its current knowledge and determine how to acquire or access any necessary additional knowledge and required updates.

Requirements regarding organizational knowledge were introduced for the purpose of safe guarding the organization from loss of knowledge, (e.g. through staff turnover; failure to capture and share information, etc.); encouraging the organization to acquire knowledge, (e.g. learning from experience; mentoring; benchmarking, etc.).

\section{Documented Information}

As shown in Table 1, one of the major differences in terminology between ISO 9001:2008 and ISO 9001:2015 was exchanging the term documentations by the new term documented information.

Where ISO 9001:2008 used specific terminology such as "document" or "documented procedures", "quality manual" or "quality plan", this edition of this International Standard defines requirements to "maintain documented information".

Where ISO 9001:2008 used the term "records" to denote documents needed to provide evidence of conformity with requirements, this is now expressed as a requirement to "retain documented information". The organization is responsible for determining what documented information needs to be retained, the

Table (1) : Major differences in terminology between ISO 9001:2008 and ISO 9001:2015.

\begin{tabular}{|l|l|}
\hline \multicolumn{1}{|c|}{ ISO 9001:2008 } & \multicolumn{1}{c|}{ ISO 9001:2015 } \\
\hline Products & Products and services \\
\hline Exclusions & $\begin{array}{l}\text { Not used } \\
\text { (See Clause A.5 for clarification of applicability) }\end{array}$ \\
\hline Management representative & $\begin{array}{l}\text { Not used } \\
\text { (Similar responsibilities and authorities are assigned } \\
\text { but no requirement for a single management repre- } \\
\text { sentative) }\end{array}$ \\
\hline $\begin{array}{l}\text { Documentation, quality manual, documented pro- } \\
\text { cedures, records }\end{array}$ & Documented information \\
\hline Work environment & Environment for the operation of processes \\
\hline Monitoring and measuring equipment & Monitoring and measuring resources \\
\hline Purchased product & Externally provided products and services \\
\hline Supplier & External provider \\
\hline
\end{tabular}


period of time for which it is to be retained and the media to be used for its retention.

\section{IAEA Safety Requirements No. GS-R-3 and GSR}

Part 2

The Safety Requirements publication titled "The Management System for Facilities and Activities" (IAEA Safety Standards Series No. GS-R-3, 2006) shows that 'Resources' are including: individuals, infrastructure, the working environment, information and knowledge, and suppliers, as well as material and financial resource (IAEA Safety Standards Series No. SF, 2006). The recently issued General Safety Requirement titled "Leadership and Management for Safety" (IAEA Safety Standards Series No. GSR Part 2, Vienna, 2016) supports the same definition of resources and in clause 4.27.,it emphasizes that organization information and knowledge should be managed as a resource (IAEA Safety Standards Series No. GS-R Part 2, 2016).

\section{IAEA Safety Guide No. GS-G-3.1}

The safety guide publication titled "Application of the Management System for Facilities and Activities", IAEA Safety Standards Series No. GS-G-3.1, IAEA, Vienna (2006) mentioned in clause no. 4.2 that using of information management, knowledge management and the corresponding technology is one of the ways that improve the performance of the organization. Also, clause 5.6 confirms that the organization should consider knowledge management to develop the processes necessary for the effective implementation of the management system (IAEA Safety Standards Series No. GS-G-3.1, 2006).

\section{IAEA Safety Guide No. GS-G-3.5}

This safety guide lists typical symptoms of a decline in safety culture in clause 2.29 and two of them are related to poor knowledge management systems, they are:

- Failure of corporate memory, which is a lack of historical data and lack of a knowledge management program to manage staff turnover. Disproportionate number of experienced individuals leaving the organization, e.g. when reorganizing and/or downsizing.

- Lack of learning, which is unwillingness to share knowledge and experience with others, or to use the experience of others to improve safety at the installation. Organizations become complacent and focus on the successes of the past, and are reluctant to invest in acquiring new knowledge and skills for the future. (IAEA Safety Standards Series No. GS-G-3.5, 2009).

\section{CONCEPT OF KNOWLEDGE}

\section{MANAGEMENT}

Knowledge management (KM) is shortly defined as the process of: capturing, developing, sharing, and effectively using organizational knowledge. It refers to a multi-disciplined approach to achieving organizational objectives by making the best use of knowledge. Broader definition of KM may be indicated in Figure 2, where wide range of possible uses or applications for KM is shown (IAEA Safety Standards Series No. GS-G-3.5, 2009).

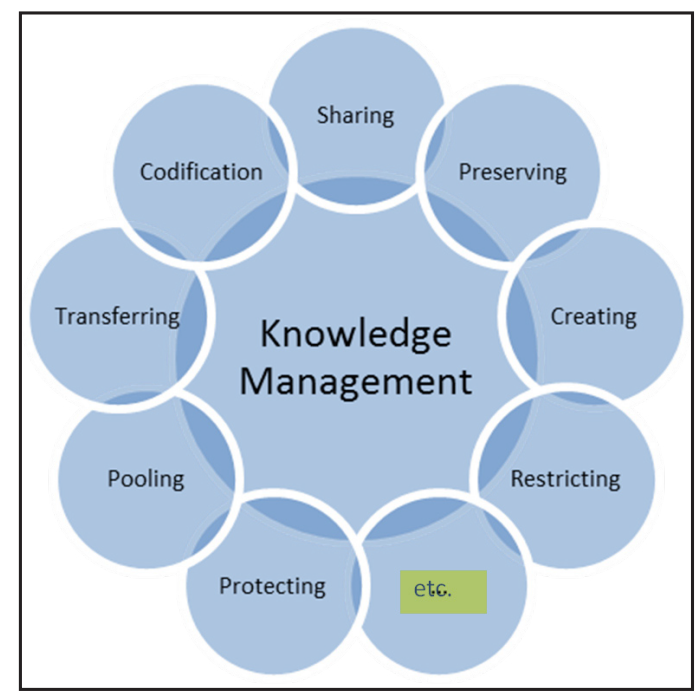

Fig. (1): Wide range of possible uses or applications for Knowledge Management. 
Knowledge management is a structured cooperation between members of the organization, technology, structure and operations of the business organization in order to add value through the reuse of knowledge or its innovative outputs. Then participation and implementation by providing the organization with valuable lessons and the best applications learned, which can be described as the memory of the organization which enables it to continue organizational learning. Generally, knowledge management focuses on:

- individuals and organizational culture to: stimulate and nurture the sharing and use of knowledge;

- processes or methods to: find, create, capture and share knowledge; and

- technology to: store knowledge and make it accessible and to allow individuals to work together without needing to be in the same place.

Individuals, specifically are considered to be the most important of these components, because managing knowledge depends mainly on the willingness of individuals to share and reuse knowledge.

\section{INTEGRATING KNOWLEDGE MANAGE-} MENT INTO EXISTING MANAGEMENT SYSTEM

A lack of nuclear safety knowledge can have significant implications much beyond an undesirable lack of efficient use of knowledge as a commercial resource. In this context, nuclear knowledge safety management is safety driven and mandatory requirement to achieve better integrated process and work management, as well as improved safety culture. Hence, effective knowledge management informs and supports the whole integrated management system process by ensuring that the right knowledge is available on the right time for the right person in order to take the right decision. One question that is often asked is "how does the organization integrate knowledge management strategies, processes and systems into the existing management system?". IAEA-TECDOC-1675 titled "Knowledge Management for Nuclear Research and Development Organizations" has answered this question as follows (IAEA-TECDOC-1675, 2012):

\section{Creating a standard for $K M$}

One useful strategy is to 'invent' a standard for $\mathrm{KM}$ based on the ISO philosophy. On this basis a KM standard would be expected to address aspects such as: Policy; Objectives and targets; Resources; Training and awareness; Communication; Controls; Monitoring and measurement; Audits and management review.

By addressing the above requirements, a systematic structure will emerge that will enable an organization's KM activities to be readily assimilated into the existing management system.

\section{High level integration options}

One approach for KM integration into the management system involves defining KM as a core process and including this as a top-level management process. This is illustrated by Fig. 3 below.

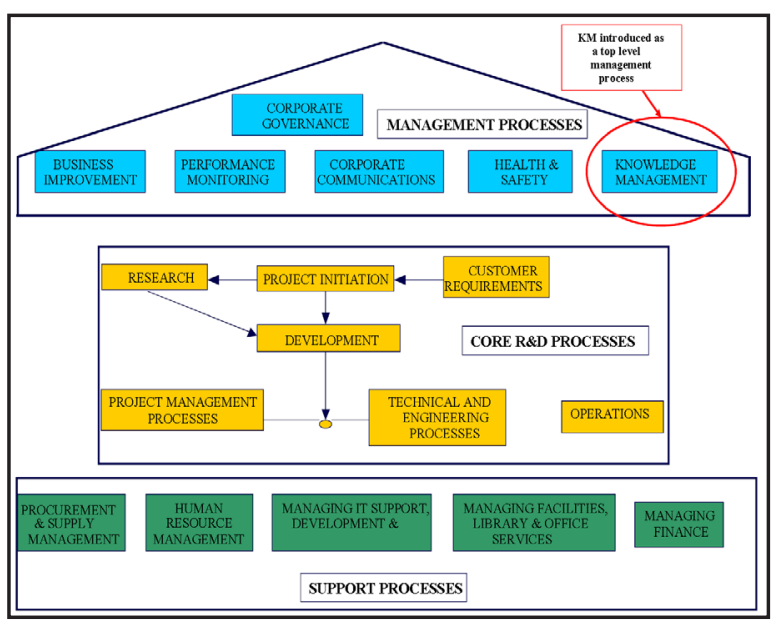

Fig. (2): Introducing KM as a major component of the management system. 
Of course, a written policy and implementation strategy for knowledge management can enhance overall effectiveness and ensure alignment with other goals, programs and processes in order to achieve a successful Integrated Management System (IMS). The main advantage of this approach is that KM is immediately elevated to a core business activity. As a central element of the management system, KM will receive attention, review and audit both internally and externally. This in turn will automatically lead to top-level management attention needed for success and further improvement (IAEA-TECDOC- 1510, 2006 - SAFETY REPORTS Series No. 75, IAEA, 2013 - Ermine, 2010).

\section{Integration at the working level}

This approach recognizes that the organization is already carrying out $\mathrm{KM}$ activities as part of its normal function but not necessarily labelling these as KM. Such activities may include knowledge transfer aspects e.g. coaching, mentoring, learning from experience, training, succession planning etc. The methodology adopted recognizes these activities form part of the organization's KM strategy but leaves them unaltered with the same ownership. The KM strategy would identify new initiatives and try to integrate these into the existing system but not at the top management level. With the ease of implementation, the transition from existing to new KM initiatives is less demanding on people's time and the resources needed.

\section{KNOWLEDGE MANAGEMENT DESIGN FOR THE ETRR 2 COMPLEX}

This section will introduce a proposal of Knowledge Management Program design for the ETRR2 complex. ETRR2 Complex contains three nuclear installations (ETRR2 Reactor, Fuel Manufacturing Pilot Plant-FMPP and Radioisotopes Production Facility-RPF) and Administrative and engineering building (headquarter of the complex), which have a fairly adequate knowledge base. This knowledge base has been preserved in the complex through the following measures:

- Its acquisition began from the design stage of the reactor as participants from EAEA were involved in the design.

- Also, participants were involved in construction and commission stages.

- During construction and commission stages, whatever possible, major tasks were photographed and video recorded.

- All basic, details, as built documents were acquired either by soft or hard copy.

- After the plant was fully operated, all work orders and working procedures were kept either in soft or hard copies.

- Major tasks were photographed and video recorded and sometimes combined with interviewing the people involved in those tasks.

- New faults and problems were written down in a log books, discussed and converted to new working procedures.

- The softcopies and video recording films were kept in a fileserver in the intranet of the complex.

- Recently, the EAEA began to send attendees to joint ICTP/IAEA NKM schools.

The main idea is to determine the performance of the KM system of the three plants (ETRR2, FMPP and RPF) and improving their function in order to avoid risks to overall operations such as 'Risk of Knowledge Loss' and lower safety and productivity. The proposal will deal with the three nuclear installations as individual projects; each project has its own team and team leader. The proposed program 
is based on the principle that the complex now has a "knowledge reservoir" of its own. This knowledge base is much more than the sum of individual knowledge of employees, and it is capitalized, more or less over time, through information products (documents, databases, software etc.) or by knowledge exchanges/transfers, individual or collective. The following steps describe the general work plan.

\section{Evaluating the existing Knowledge base}

Assessing the existing knowledge base will be achieved by evaluating and numerating documented data, information, as well as knowledge and skills of individuals. This will be in order to give a clear indication about the degree of usefulness of the existing knowledge base (Mustafa, 2012).

\section{Sources of Knowledge}

The knowledge is created by the principal "knowledge workers" of each plant most of time by interaction with the various information systems available in the complex (databases, search engines, document management systems, software etc.).Some knowledge is exchanged in an informal or semiformal way (discussions, communities, seminars etc.), it produces tacit knowledge. Some knowledge is codified in new records (publications, reports, assisting documents etc.), it is explicit knowledge. It accumulates in the firm during its history, and forms what is called a "Knowledge reservoir". Commonly, organizational knowledge is based on (Ohkubo et al., 2013):

a) internal sources: e.g. intellectual property; knowledge gained from experience; lessons learned from failures and successful tasks; capturing and sharing undocumented knowledge and experience; the results of improvements in processes, products and services; etc.

b) external sources: e.g. codes and standards; academia; conferences; technical meetings; gath- ering knowledge from external second or third party (external stakeholders or partners such as $R \& D$ entities, universities, service or product providers, etc.).

The task will be determining and numerating all these sources and collecting and classifying the available and existing data, information or knowledge from each.

\section{Used techniques to collect data}

In our approach, we used the following to document the current state (Ohkubo et al., 2013):

- Interviews: with key and knowledgeable persons such as: expertise leaders, innovation managers, senior supervisors, plant manager, etc.A questionnaire and an interview plan shall be established, a targeted group of chosen people shall be formed, according to appropriate criteria, and the analysis of the entire knowledge domain will be realized.

- Written materials: collecting, analyzing and summarizing information and documents, etc. Considering any digital asset we can treat as a structured member of a digital data base content, such as:

1. Scanned paper document

2. PDF electronics file document

3. Microsoft Office file - DOC, PPT, XLS, etc.

4. Image file - jpg, TIFF, GIF, Windows Bitmap, etc.

5. Multimedia - AVI, MPEG, Flash,

6. AutoCAD drawings,

7. Other

- Creating and updating: When it is necessary to create and update documented information, the following should be considered:

1. ensure appropriate identification and description (e.g. a title, date, author, or reference number); 
2. ensure appropriate format (e.g. language, software version, graphics) and media (e.g. paper, electronic);

3. ensure appropriate review and approval for suitability and adequacy.

- Revising and updating data of 'Resources' which includes: individuals (e.g. the number of individuals and their competences by collecting updated CVs), infrastructure, structures, systems, components, the working environment, knowledge and information, suppliers, as well as material and financial resources.

\section{SWOT Analysis}

This section is evaluating the existing knowledge base status through a SWOT analysis. As shown in figure 3, the sample S.W.O.T. analysis chart is basically standing for Strengths, Weaknesses, Opportunities, and Threats. It is almost assumed that Strengths and Weaknesses are internal factors, generated from the attributes of the organization, with a feasible control of the situation. Whereas Opportunities and Threats are generally (but not exclusively) external factors, generated from the attributes of the environment, for which there is typically a lesser level of control.

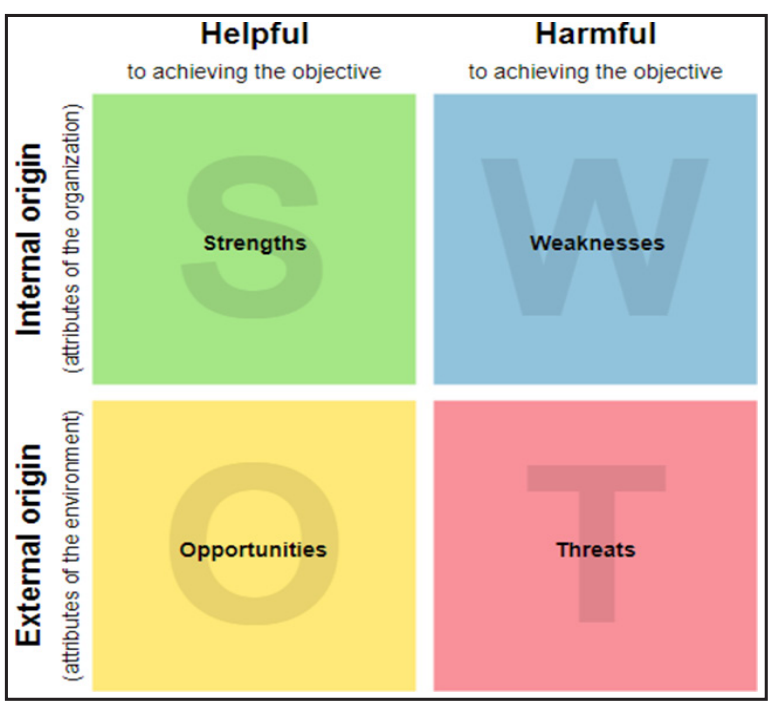

Fig. (3): Sample S.W.O.T. analysis chart.

\section{Strengths:}

Aspects which are strong abilities and advantages are:

1. Existence of advanced Hardware (LAN communication wiring, PCs, servers, etc.)

2. Existence of the technical documents for all the phases of the plants lifecycle (design, erection, commissioning, O\&M, etc.)

3. Existence of trained and licensed personnel.

4. Existence of scientists and experts of ETRR-2 Complex and EAEA.

\section{Weaknesses:}

Attention should be given to the following issues in order to ensure they are not going to be a problem in future:

1. Lack of fund for new developments / upgrading,

2. Lack of finance for new operating personnel with enough experience,

3. Bureaucratic attitude and very time-consuming administrative processes, and

4. Brain drains.

\section{Opportunities:}

The external aspects that can be usefulfor improvement:

1. Local and international markets needs of radioisotopes for medical and industrial applications (99Mo, 99mTc, 131I, 51Cr, .., 192Ir, 60Co),

2. Interest from external institutions and industry for possible services and co-operation,

3. Participation in AFRA projects and IAEA CRPs,

4. The ability of using the ETRR-2 as training center.

\section{Threats:}

The external dangers that could have a significant impact on KM system of the ETRR-2 and its facilities and need attention are: 
1. Existence of competitors (supplying radioisotopes),

2. Governmental bureaucratic procedures, and

3. Existence of job opportunities for the operating personnel in Arab and foreign countries with big difference in salaries.

\section{Implementation of KM program containing different projects}

As introduced in section 4, enhancing the existing Knowledge Base of the ETRR2 complex will be through: Establishing a set of standard principles, Integration at high and working levels, and experience drawn from others. Our references, in this case, may be: IAEA standards (IAEA-TECDOC-1675, 2012 IAEA NUCLEAR ENERGY SERIES No. NG-T6.10, 2016), our working experience, the experience of other countries having similar facilities, leading management development theorists, and design principles for each plant. In addition to this, necessary reference documentation which consists of:

- The documents on organization (organizational charts, job descriptions, descriptions of activity, portfolio of activities, etc.);

- Strategic documents (policies, missions, midterm plans, summaries of previous mid-term plans);

- Tactical and operational documents (procedures, technical and design documents, drawings, specifications, forms and records)

- Quality documents.

- Other Documents (specific reports, publications, studies, activity statements, etc.).

The purpose or business need for this program is to digitize knowledge base and infrastructures for all buildings at the same computerized data base to make a unified computerized system using server's workstation and storage station for backing up in order to reach the ideal performance of the KM system and make it a stable system. Then, make a single main storage workstation simple to access and simple to save and also to check all procedure and transfer it to multi users access template simple to print and simple to save at the same workstation which will be the main one located in the Reactor Engineering Building. The system consists of the computer hardware, software and databases that allow for the integrated preparation, input, distribution, storage, location and retrieval of electronic documents, whether initially created electronically or produced from paper documents.

\section{Project Prerequisites}

The formal inputs to the implementation proposal are:

- The vision, mission, values and objectives;

- The critical success factors;

- Relevant laws, regulations, requirements and technical codes/standards;

- Expectations of stakeholders and the internal members;

- General assessment of the current status;

- Assessment of available resources (e.g. human, financial and IT infrastructure);

- The organization's competency and knowledge. Also, for sustained benefit, cultural issues need to be addressed by assessing the current state through the answers of the following questions:

- Is knowledge sharing encouraged?

- Is knowledge regarded as critical to individual and organizational success?

- Is innovation encouraged?

- Is self/organizational learning and development 
the norm?

If the above are not in place, the project may also need to be treated as part of a cultural change initiative. It is thought that this culture is integrated with safety culture.

\section{Detailed work plan}

For each project team the following detailed plan is proposed:

- Assessing the existing Knowledge base: evaluate and numerate documented knowledge and skills of individuals based on knowledge

- Implementation of KM program containing different projects: one KM project for implementing improved KM system for each plant

- Building a team for each KM project: assigning the suitable personnel with adequate skills and experience

- Defining the KM activities and best practices such as: training (classrooms, shadow, in the job, etc.), coaching, knowledge sharing, Community of Practice CoP, Forum, etc.

Work teams will be established and composed of staff with a range of skills and abilities to help the knowledge transfer process during the project implementation phase. The project team hierarchy is shown in figure 4 and the anticipated schedule time plan and milestones is shown in figure 5 . The project deliver products or services will be infrastructure for completely computerized system and to train all who will track the system to be aware how to handle it. Also, to be aware how to keep it secured and stable system.

The idea is to follow each document in the three building (documents tracking) to make digital library for the complex. Also, to complete all document's templates (forms, checklists, ...) at the same digital stock with quality assurance procedure. This will be done using four servers one in each building of the three nuclear installations and the fourth will be the workstation for the three servers. This KM program will be flexible to accept any change to the management system infrastructure

\section{Success Factors}

Elements that are keys to the success of the project may be:

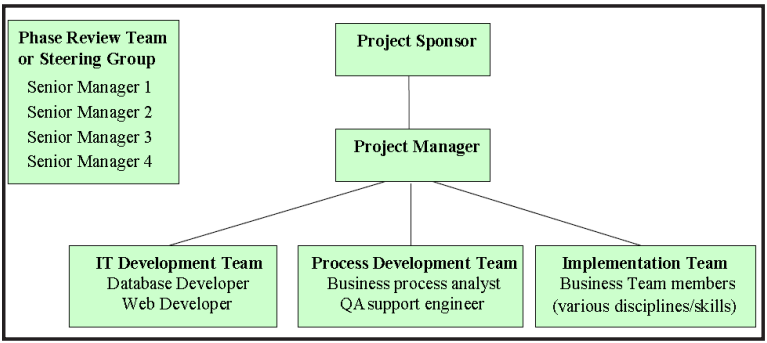

Fig. (4): Project team organizational chart.

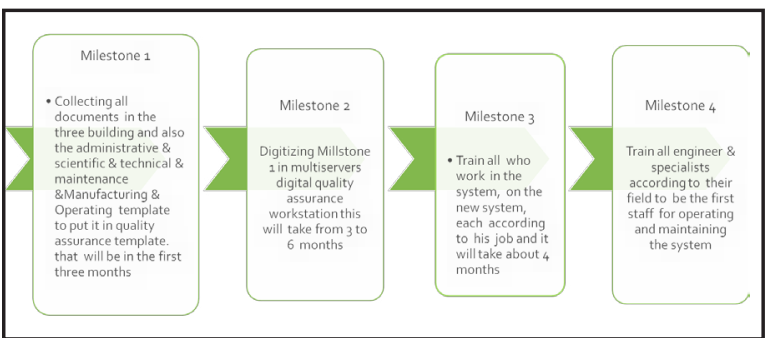

Fig. (5): Project schedule and milestone.

- to get a stable computerized KM system

- to reach satisfaction for all stakeholders

- to achieve the required information (cyber) security against any attacks

- make all administrative, scientific and technical infrastructure for the computerized system to follow the same quality assurance templates that will be handled in the system

- Delivered on time

Review of KM Projects

Project Reviews are examinations at the end of 
a project to determine if the objectives of the project were achieved; the intended benefits were realized; and also lessons can be learned for future projects. Assessment Techniques may be through:

- use of communities of Practice CoP and Forum,

- use of Online surveys,

- use of statistical frequency of using and accessing $\mathrm{CoP}$ and Forum,

- measuring the satisfaction of user through online survey and feedbacks,

- measuring the satisfaction of top-level management through set of questioners, seminars, online meetings, and video conferences,

- $\quad$ using of self-assessment and auditing (objective checklist, questionnaires to determine perception of project success), or

- Pre and post project (Key Performance Indicator) KPIs.

KPI is one of the most powerful tools in assessment and designing and generating KPIs is very challengeable issue. They are based mainly on feedback from employees (engineers, scientists, workers, management, etc.), as well as stakeholders. They may include:

- Customer Satisfaction: The customers fill a feedback form where they indicate clearly their level of satisfaction with the service and the value of the recommendations to the business

- On-time completion of projects: Every time a project is completed, the systems compares the planned and actual completion dates and decides whether the project is late or completed on-time.

- Business impact, showing the effect of the system to save money to the complex: Business and technical staff agree on a business impact number for that specific technical project as it relates to the KM system.
- The total number of technical reports generated per year, showing accumulation of knowledge wealth over time: The KM system should have the ability to records automatically the number of technical reports, and archives them in a predesigned taxonomy in the knowledge base.

Statistical figures for these KPI may be generated and logged. This gives a clear indication about the usefulness of the KM system for the complex.

\section{Issues and challenges related to KM establishment and how to overcome them}

This section is summarizing issues and challenges related to KM establishment and how to overcome them. The most common issues and challenges are within the following:

- Cyber Security.

- IT Difficulties.

- Laws and Regulations.

- Security Clearances.

- $\quad$ Lake of resources (5 Ms):

1. Money (Funds and Budget).

2. Man (personnel and expertise)

3. Machine (necessary SSCs)

4. Material (Knowledge)

5. Management (commitment and attitude)

There are some ideas to overcome those challenges, such as:

- Just by integrating the KM system within the IMS would already help

- More informal ways of improvement actions: Observations - Witnessing

- Small rewards (not even money, but any kind of reward) 
- Follow-up actions, reviews, reporting to find out if recommendations have been fulfilled

- If actions are not fulfilled find out why, find root causes and work on them

- KPI's on "action-efficiency" to top management

- Practical regional training workshop KM

- Practical examples, success stories about integrating $\mathrm{KM}$ within management systems

- Fellowship on practical issues of the improvement cycle like self-assessment, observations, technical cooperation adapted to the size and power of the installation, Web Forum, Platform and good practices on KM for nuclear installations.

- New laws and regulations should be issued.

- More I.T. staff should be provided to the complex to support KM.

Of course, when knowledge management tools and techniques are generally integrated into the quality management system as formally written processes, approaches such as self-assessments for knowledge management and benchmarking will be used. In this case, knowledge management system including work activities and operational experience processes, findings and lessons learned well be documented and communicated.

\section{SUMMERY AND CONCLUSSION}

This paper introduces a proposal of Knowledge Management Program design for the ETRR2 complex. ETRR2 Complex contains three nuclear installations (ETRR2 Reactor, Fuel Manufacturing Pilot Plant-FMPP and Radioisotopes Production FacilityRPF) and Administrative and engineering building (headquarter of the complex), which have a fairly adequate knowledge base. This knowledge base is much more than the sum of individual knowledge of employees; it is capitalized, more or less over time, through information products (documents, databases, software etc.) or by knowledge exchanges/ transfers, individual or collective. The main idea is to determine the performance of the KM system of the three plants (ETRR2, FMPP and RPF) and improving their function in order to avoid risks to overall operations such as 'Risk of Knowledge Loss' and lower safety and productivity. The proposal deals with the three nuclear installations as individual projects; each project has its own team and team leader. The proposed program is based on the principle that the complex now has a "knowledge reservoir" of its own and it should be utilized in order to gain the following advantages:

- To give a clear indication about the usefulness of the existing knowledge base.

- Making available increased knowledge content in the development and provision of high levels of performance.

- Improving the human resources and technology management

- achieving organizational objectives by making the best use of knowledge

- achieving greater shearing of human and other resources among ETRR2 facilities

- Encourage teamwork to improve performance

- Aid in achieving the production and safety potentials of the complex

- Functions are shared between several senior experts

- Very important step in the process of applying the IMS.

In this respect Knowledge Management is fundamental, and initiating a KM program involving all the buildings of the complex is highly desirable in order to capture, retain and distribute knowledge, 
while helping prevent its loss. To achieve this in an efficient manner, KM processes and tools need to be assimilated into the organization structure and become part of the organizational culture and the daily operational processes, aiming towards an integrated management system.

\section{REFERENCES}

- Ermine, J. (2010): Methods and tools for knowledge management in research centers, Electronic Journal of Knowledge Management, 8(3): 293.

- IAEA-TECDOC- 1510. (2006): Knowledge Management for Nuclear Industry Operating Organizations, International Atomic Energy Agency, Vienna.

- IAEA-TECDOC-1675. (2012): Knowledge management for nuclear research and development organizations, International Atomic Energy, Vienna.

- ISO 9000. (2015): Quality management system: fundamentals and vocabulary, $4^{\text {th }}$ edition 9:15.

- ISO 9001. (2015): Quality management system requirements, fifth edition 9:15.

- Mustafa, M.M.T (2012): A Model of integration between knowledge management and business processes of industrial small and medium-sized enterprises. Int. J. Database Manag. Syst., 4(4): 13.

- Nuclear Energy Series No. NG-T-6.10. (2016): Knowledge management and its implementation in nuclear organizations, International Atomic Energy Agency, Vienna.

- Ohkubo, S.; Sullivan. T.M.; Harlan, S.V.; Timmons, B.K. and Strachan, M. (2013): Guide to monitoring and evaluating knowledge management in global health programs, Baltimore, MD: Center for Communication Programs, Johns Hopkins Bloomberg School of Public Health.

- Safety Reports Series No. 75. (2013): Implementation of a management system for operating organizations of research reactors, International Atomic Energy Agency, Vienna.
- Safety Standards Series No. GS-G-3.1. (2006): Application of the management system for facilities and activities, International Atomic Energy Agency, Vienna.

- Safety Standards Series No. GS-G-3.5. (2009): The management system for nuclear installations, International Atomic Energy Agency, Vienna.

- Safety Standards Series No. GS-R Part 2. (2016): Leadership and management for safety, International Atomic Energy, Vienna.

- Safety Standards Series No. GS-R-3. (2006): The safety requirements publication the management system for facilities and activities, International Atomic Energy Agency, Vienna.

- Safety Standards Series No. SF. (2006): Fundamental safety principles, International Atomic Energy Agency, Vienna. 
